\title{
MINIMAX-OPTIMAL STRATEGIES FOR THE BEST-CHOICE PROBLEM WHEN A BOUND IS KNOWN FOR THE EXPECTED NUMBER OF OBJECTS*
}

\author{
T. P. HILL ${ }^{\dagger}$ AND D. P. KENNEDY ${ }^{\ddagger}$
}

\begin{abstract}
For the best-choice (or secretary) problem with an unknown number $N$ of objects, minimax-optimal strategies for the observer and minimax distributions for $N$ are derived under the assumption that $N$ is a random variable with expected value at most $M$, where $M$ is known. The solution is derived as a special case of the situation where $N$ is constrained by $E f(N) \leq M$, where $f$ is increasing with $f(i)-f(i-1)$ convex.
\end{abstract}

Key words. best-choice problem; secretary problem; minimax strategies; optimal stopping; convexity; Lagrangian; Lagrange multiplier

AMS subject classification. $60 \mathrm{G} 40$

1. Introduction. In the classical best-choice (or secretary) problem a known fixed number, $n$, of rankable objects is presented one by one in random order (all $n$ ! possible orderings being equally likely). As each object is presented, the observer must either select it and stop observing or reject it and continue observing. He may never return to a previously rejected object, and his decision to stop must be based solely on the relative ranks of the objects he has observed so far. The goal is to maximize the probability that the best object is selected. For a history and review of the literature of this problem and its numerous variants the reader is referred to Freeman (1983) and Ferguson (1989). In the best-choice setting, the optimal strategy for the observer is to view $k_{n}$ objects without selecting and subsequently to take the first object, if any, better than all its predecessors, where $k_{1}=0$ and for $n>1, k_{n}$ is the unique positive integer satisfying

$$
\sum_{i=k_{n}}^{n-1} \frac{1}{i} \geq 1>\sum_{i=k_{n}+1}^{n-1} \frac{1}{i} .
$$

If the number of objects is not known, but is a random variable taking values in the positive integers, then minimax-optimal strategies for selecting the best object are known for several situations (cf. Freeman (1983), Ferguson (1989)). For example, Presman and Sonin (1972) derived the optimal stop rules when the distribution of $N$ is known, and Hill and Krengel (1991) found minimax-optimal stop rules (and distributions) when $N$ has unknown distribution but known upper bound $n$. It is the purpose of this paper to derive the analogous minimax-optimal strategies when $N$ again has unknown distribution, but has expectation at most $M$, where $M$ is known. Since the arguments in this case generalize easily to the constraint $E f(N) \leq M$, where $f$ is a known positive function for which $f(i)-f(i-1)$ is nondecreasing and convex, the proofs will be given in the more general setting. The reader may want to keep in mind the natural case $f(i) \equiv i$, which corresponds to the expected-value constraint.

In the (zero-sum, two-person) game-theoretic interpretation of this problem there are two players, a controller $P$ and an observer $Q$. Given $M>0$ and a constraint function $f$, player $P$ first picks a distribution for the number of objects, subject to the constraint $E(f(N)) \leq M$, and then the actual number $N$ of objects to be presented to $Q$ is chosen randomly according to this distribution. Then, knowing only the constraint (and not $N$ itself), player $Q$ begins

\footnotetext{
* Received by the editors July 23, 1992; accepted for publication (in revised form) January 7, 1993.

$\dagger$ Department of Mathematics, Georgia Institute of Technology, Atlanta, Georgia 30332. The work of this author was partially supported by National Science Foundation grant DMS 89-01267.

$\ddagger$ Statistical Laboratory, Department of Pure Mathematics and Mathematical Statistics, University of Cambridge, 16 Mill Lane, Cambridge CB2 1SB, United Kingdom.
} 
his observation-selection of the objects and receives one dollar from player $P$ if the object he selects is the best of the $N$ objects and pays player $Q$ one dollar if it is not the best.

Formally, the strategies available to the two players are given as follows. For $M \geq f(1)$, the set of allowable strategies for player $P$ is

$$
\mathcal{P}_{M}=\left\{\mathbf{p}=\left(p_{1}, p_{2}, \cdots\right): p_{j} \geq 0, \sum_{j=1}^{\infty} p_{j}=1, \sum_{j=1}^{\infty} f(j) p_{j} \leq M\right\}
$$

(the set of distributions for $N$ for which $E f(N) \leq M$ ), and

$$
\mathcal{Q}=\left\{\mathbf{q}=\left(q_{1}, q_{2}, \cdots\right): 0 \leq q_{j} \leq 1\right\}
$$

is the set of allowable strategies for player $Q$, where if strategy $\mathbf{q}$ is used player $Q$ stops at object $j$ with probability $q_{j}$ (independently of the rest of the process) if object $j$ is the best so far. If the strategies $\mathbf{p}, \mathbf{q}$ are used by the respective players, the pay-off function $V(\mathbf{p}, \mathbf{q})$, which is the probability that player $Q$ selects the best object, is given (cf. Hill and Krengel (1991)) by

$$
V(\mathbf{p}, \mathbf{q})=\sum_{j=1}^{\infty} \frac{p_{j}}{j} \sum_{i=1}^{j} q_{i} \prod_{m=1}^{i-1}\left(1-\frac{q_{m}}{m}\right) .
$$

For each value of $M \geq f(1)$, the aim is to derive minimax-optimal strategies $\mathbf{p}_{M} \in \mathcal{P}_{M}$, $\mathbf{q}_{M} \in \mathcal{Q}$ satisfying

$$
V\left(\mathbf{p}_{M}, \mathbf{q}\right) \leq V\left(\mathbf{p}_{M}, \mathbf{q}_{M}\right) \leq V\left(\mathbf{p}, \mathbf{q}_{M}\right) \quad \text { for all } \mathbf{p} \in \mathcal{P}_{M}, \mathbf{q} \in \mathcal{Q}
$$

Example 1.1. As will follow from the main results below, for the optimal strategies when $N$ is a random variable with expected value at most 3 , the optimal strategy for the observer is $\left(\frac{7}{13}, 1,1, \cdots\right)$, i.e., stop with the first object with probability $\frac{7}{13}$, and otherwise stop with the first object thereafter, if any, that is better than any previously seen. Using this strategy, the best object will be selected with probability at least $\frac{16}{39}$ no matter what the distribution of $N$ is, provided its expectation is at most 3. Conversely, the optimal $P$-strategy (worst-case distribution) for this case is $\left(\frac{2}{13}, 0, \frac{7}{13}, \frac{4}{13}, 0,0, \cdots\right)$, i.e., there is only the one object with probability $\frac{2}{13}$, exactly three objects with probability $\frac{7}{13}$, and exactly four objects with probability $\frac{4}{13}$; and against this distribution no stop rule will select the best object with probability exceeding $\frac{16}{39}$.

That the optimal $Q$-strategy is monotonic is intuitive (since if it is good to stop at time $j$ with the best object seen so far, it is even better to stop at later times if that object is the best yet seen), but that the optimal $P$-strategy typically (as in Example 1.1) places mass on two large numbers seems surprising, and it is never the case for the uniformly bounded problem. In general, the optimal value is a complicated piecewise linear function of the form $\lambda+\mu M$ for appropriate $\lambda$ and $\mu$. It will be seen that there are real numbers $a_{1}<a_{2}<\cdots$, such that the minimax-optimal $\mathbf{q}_{M}$ is constant over each interval $a_{i} \leq M \leq a_{i+1}$, while the minimaxoptimal $\mathbf{p}_{M}$ is linear in $M$ in the interval and is thus a convex combination of the distributions that are optimal at the end points $M=a_{i}$ and $M=a_{i+1}$. The next example, which identifies values and optimal strategies for an interval of values of $M$ (including the special case $M=3$ of Example 1.1), shows typical behavior of the optimal strategies and value as $M$ varies.

Example 1.2. As will follow from the main results below, for the expected-value constraint $f(i) \equiv i$, the optimal strategies and value for $19 / 7 \leq M \leq 317 / 75$ are as follows. 
If $19 / 7 \leq M<101 / 29$ :

$$
\begin{aligned}
& \mathbf{q}_{M}=\left(\frac{7}{13}, 1,1, \cdots\right) \\
& \mathbf{p}_{M}=a_{M}\left(\frac{1}{7}, 0, \frac{6}{7}, 0,0, \cdots\right)+\left(1-a_{M}\right)\left(\frac{5}{29}, 0,0, \frac{24}{29}, 0,0, \cdots\right),
\end{aligned}
$$

where $a_{M}=7(101-29 M) / 156$;

$$
V\left(\mathbf{p}_{M}, \mathbf{q}_{M}\right)=(47-5 M) / 78 .
$$

If $101 / 29 \leq M<69 / 17$ :

$$
\begin{aligned}
& \mathbf{q}_{M}=\left(\frac{105}{213}, 1,1, \cdots\right) \\
& \mathbf{p}_{M}=b_{M}\left(\frac{5}{29}, 0,0, \frac{24}{29}, 0, \cdots\right)+\left(1-b_{M}\right)\left(\frac{3}{17}, 0,0, \frac{4}{17}, \frac{10}{17}, 0, \cdots\right)
\end{aligned}
$$

where $b_{M}=29(69-17 M) / 284$;

$$
V\left(\mathbf{p}_{M}, \mathbf{q}_{M}\right)=(459-39 M) / 852 .
$$

If $69 / 17 \leq M<317 / 75$ :

$$
\begin{aligned}
& \mathbf{q}_{M}=\left(\frac{50}{107}, \frac{14}{19}, 1,1, \cdots\right) \\
& \mathbf{p}_{M}=c_{M}\left(\frac{3}{17}, 0,0, \frac{4}{17}, \frac{10}{17}, 0,0, \cdots\right)+\left(1-c_{M}\right)\left(\frac{13}{75}, \frac{2}{75}, 0,0, \frac{60}{75}, 0, \cdots\right),
\end{aligned}
$$

where $c_{M}=17(317-75 M) / 214$;

$$
V\left(\mathbf{p}_{M}, \mathbf{q}_{M}\right)=(54-4 M) / 107
$$

Although the statements of the main results in this article are probabilistic in nature, the proofs are primarily optimization-theoretic. Since general optimization theory saddle-point theorems do not seem to yield a direct solution to the problem formulated here, optimization arguments using a Lagrangian, but heavily based on ad hoc convexity tools, have been developed. In principle, one could use the same techniques to handle a larger class of constraint functions such as those reflecting known bounds on means and variances (or several other moments), but this would involve examination of the many cases corresponding to criticality of the various constraints and is not done here.

The organization of the paper is as follows. Section 2 introduces notation, important parameters, and the value of the game and establishes a number of useful identities and inequalities, the proofs of which may be skipped at first reading. Section 3 identifies the minimax-optimal strategy for the observer player $Q$, which is obtained by solving for the coefficient of $p_{j} / j$ in a Lagrangian, and $\S 4$ builds on these results to establish the optimal (worst-case) distribution for $N$ (i.e., the minimax-optimal strategy for the controller player $P$ ), and summarizes all the results in the main theorem, Theorem 4.3. 
2. Notation, preliminaries and the value of the game. The first lemma records some easy convexity results; the proof is left to the reader.

LEMMA 2.1. Suppose $g: \mathbb{N} \rightarrow(0, \infty)$. Then

(i) $g$ is convex (respectively, strictly convex) if and only if $g(i)-g(i-1)$ is nondecreasing (increasing) in $i$

(ii) if $g$ is convex (respectively, strictly convex), then $\sum_{j=k+1}^{i} g(j) /(i-k)$ is convex (strictly convex) in $i>k$ for each $k \geq 0$.

Basic assumption. Throughout this paper, $f(0)=0$ and $f: \mathbb{N} \rightarrow(0, \infty)$ and

$$
f(i)-f(i-1) \text { is nondecreasing and convex, }
$$

the canonical example being $f(i) \equiv i$.

Let $s_{0}=0, s_{k}=\sum_{j=1}^{k} 1 / j$ for $k \geq 1$ and for $1 \leq k<n$, set $s_{k}^{n}=s_{n}-s_{k}$ and $F_{k}^{n}=n f(n)-k f(k)$.

LEMMA 2.2. For all such $f$,

(i) $f(i)$ is increasing and convex on $i \in \mathbb{N}$;

(ii) if $(i)$ is increasing and strictly convex on $i \in \mathbb{N}$;

(iii) if $(i)-(i-1) f(i-1)$ is increasing and convex on $i \in \mathbb{N}$;

(iv) $s_{k}^{n} /(n-k)$ is decreasing and strictly convex in $n>k$;

(v) $F_{k}^{n} /(n-k)$ is increasing and convex in $n>k$;

(vi) $F_{k}^{n} / s_{k-1}^{n-1}$ is increasing in $n>k$,

(vii) $\frac{F_{k}^{n+1}}{n-k+1}>\frac{F_{k}^{n}}{n-k} \geq F_{k}^{k+1}>f(k)>\left(\sum_{j=1}^{k-1} \frac{f(j)}{j+1}+f(k)\right) / s_{k}$.

Proof. The proof is routine, using (3), Lemma 2.1 and the definitions of $s_{k}^{n}$ and $F_{k}^{n}$.

The next objective will be to define some basic parameters that play a central role in the main results of this paper and to establish some useful inequalities and equalities interrelating these parameters.

For $1 \leq k<n$, define $\alpha_{n, k}>0, \bar{\alpha}_{n, k}>0, m_{n, k}>0, \bar{m}_{n, k}>0, \lambda_{n, k}>0$, and $\mu_{n, k}<0$, as follows:

$$
\frac{1}{\alpha_{n, k}}=s_{k}+\frac{n-k}{k s_{k-1}^{n-1}}, \quad \frac{1}{\bar{\alpha}_{n, k}}=s_{k}+\frac{n-k+n\left(1-s_{k}^{n-1}\right)}{k+1},
$$

$$
\begin{aligned}
\frac{m_{n, k}}{\alpha_{n, k}} & =\sum_{j=1}^{k-1} \frac{f(j)}{j+1}+f(k)+\frac{F_{k}^{n}}{k s_{k-1}^{n-1}} \\
\frac{\bar{m}_{n, k}}{\bar{\alpha}_{n, k}} & =\sum_{j=1}^{k-1} \frac{f(j)}{j+1}+f(k)+\frac{F_{k}^{n}+n F_{n}^{n+1}\left(1-s_{k}^{n-1}\right)}{k+1}
\end{aligned}
$$

$$
\lambda_{n, k}=\frac{\alpha_{n, k} m_{n+1, k}-\alpha_{n+1, k} m_{n, k}}{m_{n+1, k}-m_{n, k}}, \quad \mu_{n, k}=\frac{\alpha_{n+1, k}-\alpha_{n, k}}{m_{n+1, k}-m_{n, k}} .
$$

Note that $\alpha_{n, k}$ and $\bar{\alpha}_{n, k}$ do not depend on $f$. Using these parameters, the value of the game $\mathbf{v}_{M}=V\left(\mathbf{p}_{M}, \mathbf{q}_{M}\right)$ appearing in (2) can now be stated (although proof that it is indeed the value is the subject of the subsequent sections).

Recall that $k_{n}$ is the optimal cutoff value for the classical secretary problem with $n$ objects and so $s_{k_{n}-1}^{n-1} \geq 1>s_{k_{n}}^{n-1}$ and $k_{n} \leq k_{n+1} \leq k_{n}+1$. Set $m_{n}=m_{n, k_{n}}, \bar{m}_{n}=\bar{m}_{n, k_{n}}, \alpha_{n}=$ $\alpha_{n, k_{n}}$, and $\bar{\alpha}_{n}=\bar{\alpha}_{n, k_{n}}$. 
DEFINITION. For all $M>f(1)$,

$$
v_{M}= \begin{cases}\lambda_{n, k_{n}}+\mu_{n, k_{n}} M & \text { if } m_{n} \leq M<m_{n+1} \text { and } k_{n+1}=k_{n}, \text { or } \\ & \text { if } m_{n} \leq M<m_{n} \text { and } k_{n+1}=k_{n}+1 \\ \lambda_{n, k_{n+1}}+\mu_{n, k_{n+1}} M \quad \text { if } \bar{m}_{n} \leq M<m_{n+1} \text { and } k_{n+1}=k_{n}+1\end{cases}
$$

Notice that $v_{m_{n}}=\alpha_{n}$, and $v_{\bar{m}_{n}}=\bar{\alpha}_{n}$ when $k_{n+1}=k_{n}+1$.

Example 2.3. For the canonical expected-value case $f(i) \equiv i$,

$$
\begin{gathered}
\left(m_{3}, m_{4}, \bar{m}_{4}, m_{5}\right)=\left(\frac{19}{7}, \frac{101}{29}, \frac{69}{17}, \frac{317}{75}\right), \\
\left(\alpha_{3,1}, \alpha_{4,1}, \alpha_{5,1}, \alpha_{4,2}, \alpha_{5,2}\right)=\left(\frac{3}{7}, \frac{11}{29}, \frac{25}{73}, \frac{10}{27}, \frac{26}{75}\right), \\
\left(m_{3,1}, m_{4,1}, m_{5,1}, m_{4,2}, m_{5,2}\right)=\left(\frac{19}{7}, \frac{101}{29}, \frac{313}{73}, \frac{97}{27}, \frac{317}{75}\right), \\
\left(\lambda_{3,1}, \lambda_{4,1}, \lambda_{4,2}\right)=\left(\frac{47}{78}, \frac{459}{852}, \frac{54}{107}\right), \text { and } \\
\left(\mu_{3,1}, \mu_{4,1}, \mu_{4,2}\right)=\left(\frac{-5}{78}, \frac{-39}{852}, \frac{-4}{107}\right),
\end{gathered}
$$

and together with (7) these yield the value $v_{M}=V\left(\mathbf{p}_{M}, \mathbf{q}_{M}\right)$ in Example 1.2.

The next lemma establishes some useful inequalities.

LEMMA 2.4. For $n>1$,

(i) for fixed $k, \alpha_{n, k}$ is decreasing in $n>k$;

(ii) for fixed $k, m_{n, k} / \alpha_{n, k}$ and $m_{n, k}$ are increasing in $n>k$;

(iii) for fixed $n, \alpha_{n, k}$ is maximized at $k=k_{n} ; \alpha_{n, k}$ is increasing in $k, 1 \leq k \leq k_{n}$, and decreasing in $k, k_{n} \leq k \leq n$;

(iv) for fixed $n, m_{n, k} / \alpha_{n, k}$ and $m_{n, k}$ are minimized at $k=k_{n}$; they are decreasing in $k, 1 \leq k \leq k_{n}$ and increasing in $k, k_{n} \leq k \leq n$;

(v) $\bar{\alpha}_{n}<\alpha_{n}<\alpha_{n-1}$

(vi) $\bar{m}_{n}>m_{n}>m_{n-1}$;

(vii) $\bar{\alpha}_{n} \geq \alpha_{n+1}$ when $k_{n+1}=k_{n}+1$;

(viii) $m_{n, k_{n+1}} \leq \bar{m}_{n} \leq m_{n+1}<m_{n+1, k_{n}}$ when $k_{n+1}=k_{n}+1$; and

(ix) $\lambda_{n, k}>0$ and $\mu_{n, k}<0$.

Proof. (i) By Lemma 2.2 (iv),

$$
1 / \alpha_{n, k}=s_{k}+(n-k) /\left(k s_{k-1}^{n-1}\right)<s_{k}+(n-k+1) /\left(k s_{k-1}^{n}\right)=1 / \alpha_{n+1, k} .
$$

(ii) By Lemma 2.2 (vi),

$$
\frac{m_{n+1, k}}{\alpha_{n+1, k}}-\frac{m_{n, k}}{\alpha_{n, k}}=\frac{F_{k}^{n+1}}{k s_{k-1}^{n}}-\frac{F_{k}^{n}}{k s_{k-1}^{n-1}}>0 .
$$

Also, (i), (4), and Lemma 2.2 (vii) give

$$
\begin{aligned}
m_{n+1, k}-m_{n, k}= & \left(\alpha_{n, k}-\alpha_{n+1, k}\right)\left(\frac{s_{k} F_{k}^{n}}{n-k}-\sum_{j=1}^{k-1} \frac{f(j)}{j+1}-f(k)\right) \\
& +\left(\frac{F_{k}^{n+1}}{n-k+1}-\frac{F_{k}^{n}}{n-k}\right)\left(1-s_{k} \alpha_{n+1, k}\right)>0 .
\end{aligned}
$$


(iii) Observing that $n-k-1>k s_{k}^{n-1}$, it may be seen that the difference

$$
\frac{1}{\alpha_{n, k+1}}-\frac{1}{\alpha_{n, k}}=\frac{\left(s_{k}^{n-1}-1\right)\left(1-(n-k-1) /\left(k s_{k}^{n-1}\right)\right)}{(k+1) s_{k-1}^{n-1}}
$$

is $\leq 0$ or $>0$ according as $k<k_{n}$ or $k \geq k_{n}$.

(iv) By Lemma 2.2 (ii), $F_{k+1}^{n}>(n-k-1) F_{k}^{k+1}$ and since $n-k-1>k s_{k}^{n-1}$,

$$
\frac{F_{k+1}^{n}}{k s_{k}^{n-1}}=\left(\frac{F_{k+1}^{n}}{n-k-1}\right)\left(\frac{n-k-1}{k s_{k}^{n-1}}\right)>F_{k}^{k+1} \text {. }
$$

It follows that

$$
\frac{m_{n, k+1}}{\alpha_{n, k+1}}-\frac{m_{n, k}}{\alpha_{n, k}}=\frac{\left(s_{k}^{n-1}-1\right)}{(k+1) s_{k-1}^{n-1}}\left(F_{k}^{k+1}-\frac{F_{k+1}^{n}}{k s_{k}^{n-1}}\right)
$$

is $\leq 0$ or $>0$ according as $k<k_{n}$ or $k \geq k_{n}$. This last equality implies

$$
\begin{aligned}
m_{n, k+1}-m_{n, k} & =\alpha_{n, k+1}\left[\frac{m_{n, k+1}}{\alpha_{n, k+1}}-\frac{m_{n, k}}{\alpha_{n, k}}+m_{n, k}\left(\frac{1}{\alpha_{n, k}}-\frac{1}{\alpha_{n, k+1}}\right)\right] \\
& =\frac{\alpha_{n, k+1}\left(s_{k}^{n-1}-1\right)}{(k+1) s_{k-1}^{n-1}}\left[F_{k}^{k+1}-m_{n, k}+\left(\frac{(n-k-1) m_{n, k}-F_{k+1}^{n}}{k s_{k}^{n-1}}\right)\right] .
\end{aligned}
$$

From (4), (5), and Lemma 2.2 (vii) note that

$$
\frac{m_{n, k}}{\alpha_{n, k}}<s_{k} f(k)+\frac{F_{k}^{n}}{k s_{k-1}^{n-1}}=s_{k}\left(f(k)-\frac{F_{k}^{n}}{n-k}\right)+\frac{F_{k}^{n}}{(n-k) \alpha_{n, k}}<\frac{F_{k}^{n}}{(n-k) \alpha_{n, k}}
$$

hence

$$
m_{n, k}<\frac{F_{k}^{n}}{n-k}<\frac{F_{k+1}^{n}}{n-k-1}<F_{n-1}^{n}
$$

Since $n-k-1>k s_{k}^{n-1}$, it follows that

$$
F_{k}^{k+1}-m_{n, k}+\left(\frac{(n-k-1) m_{n, k}-F_{k+1}^{n}}{k s_{k}^{n-1}}\right)<F_{k}^{k+1}-\frac{F_{k+1}^{n}}{n-k-1}<0,
$$

which implies that $m_{n, k+1} \leq m_{n, k}$ or $m_{n, k+1}>m_{n, k}$ according as $k<k_{n}$ or $k \geq k_{n}$.

(v) Note that $n s_{k-1}^{n-1}>n-k$. Since from (4),

$$
\frac{1}{\bar{\alpha}_{n, k}}-\frac{1}{\alpha_{n, k}}=\frac{\left(1-s_{k}^{n-1}\right)\left(n s_{k-1}^{n-1}-(n-k)\right)}{(k+1) s_{k-1}^{n-1}},
$$

putting $k=k_{n}$ and recalling the fact that $1>s_{k_{n}}^{n-1}$ gives the result.

(vi) From (5),

$$
\frac{\bar{m}_{n, k}}{\bar{\alpha}_{n, k}}-\frac{m_{n, k}}{\alpha_{n, k}}=\frac{\left(1-s_{k}^{n-1}\right)}{k+1}\left(n F_{n}^{n+1}-\frac{F_{k}^{n}}{s_{k-1}^{n-1}}\right) .
$$


Hence

$$
\begin{aligned}
\bar{m}_{n, k}-m_{n, k} & =\bar{\alpha}_{n, k}\left[\frac{\bar{m}_{n, k}}{\bar{\alpha}_{n, k}}-\frac{m_{n, k}}{\alpha_{n, k}}+m_{n, k}\left(\frac{1}{\alpha_{n, k}}-\frac{1}{\bar{\alpha}_{n, k}}\right)\right] \\
& =\frac{\bar{\alpha}_{n, k}\left(1-s_{k}^{n-1}\right)}{k+1}\left[n\left(F_{n}^{n+1}-m_{n, k}\right)-\frac{F_{k}^{n}-(n-k) m_{n, k}}{s_{k-1}^{n-1}}\right] .
\end{aligned}
$$

Again using the inequality $s_{k-1}^{n-1}>(n-k) / n$ and (8),

$$
n\left(F_{n}^{n+1}-m_{n, k}\right)-\frac{F_{k}^{n}-(n-k) m_{n, k}}{s_{k-1}^{n-1}}>n\left(F_{n}^{n+1}-\frac{F_{k}^{n}}{n-k}\right)>0,
$$

whence $\bar{m}_{n, k}-m_{n, k}$ is $>0$ or $\leq 0$ according as $1>s_{k}^{n-1}$ or $1 \leq s_{k}^{n-1}$. From (ii) and (iv) above, $m_{n}=m_{n, k_{n}} \leq m_{n, k_{n+1}}<m_{n+1, k_{n+1}}=m_{n+1}$.

(vii) Similarly,

$$
\frac{1}{\bar{\alpha}_{n, k}}-\frac{1}{\alpha_{n+1, k+1}}=\frac{\left(1-s_{k}^{n}\right)\left(n s_{k}^{n}-(n-k)\right)}{(k+1) s_{k}^{n}},
$$

which is $\leq 0$ or $>0$ according as $k<k_{n+1}$ or $k \geq k_{n+1}$; taking $k=k_{n}<k_{n+1}$ gives the result.

(viii) Note that

$$
\frac{m_{n+1, k+1}}{\alpha_{n+1, k+1}}-\frac{\bar{m}_{n, k}}{\bar{\alpha}_{n, k}}=\frac{\left(1-s_{k}^{n}\right)\left(F_{k+1}^{n+1}-n F_{n}^{n+1} s_{k}^{n}\right)}{(k+1) s_{k}^{n}},
$$

and it follows using the expression in the proof of (vii) above that

$$
\begin{aligned}
m_{n+1, k+1}-\bar{m}_{n, k} & =\bar{\alpha}_{n, k}\left[\frac{m_{n+1, k+1}}{\alpha_{n+1, k+1}}-\frac{\bar{m}_{n, k}}{\bar{\alpha}_{n, k}}+m_{n+1, k+1}\left(\frac{1}{\bar{\alpha}_{n, k}}-\frac{1}{\alpha_{n+1, k+1}}\right)\right] \\
& =\frac{\bar{\alpha}_{n, k}\left(1-s_{k}^{n}\right)}{(k+1) s_{k}^{n}}\left[F_{k+1}^{n+1}-(n-k) m_{n+1, k+1}-n s_{k}^{n}\left(F_{n}^{n+1}-m_{n+1, k+1}\right)\right] .
\end{aligned}
$$

Observe from (8) that $m_{n+1, k+1}<F_{n}^{n+1}$ and using $n s_{k}^{n}>n-k$ gives

$$
F_{k+1}^{n+1}-(n-k) m_{n+1, k+1}-n s_{k}^{n}\left(F_{n}^{n+1}-m_{n+1, k+1}\right)<0,
$$

from which it may be seen that $\bar{m}_{n} \leq m_{n+1}<m_{n+1, k_{n}}$ by setting $k=k_{n}<k_{n+1}$ and using (iv) above. That $m_{n, k_{n+1}} \leq \bar{m}_{n}$ in this case follows in a similar fashion.

(ix) The conclusion follows easily from (6) and (i)-(viii) above.

The next lemma records some useful identities relating the parameters.

LEMMA 2.5. For $1 \leq k<n$, the following equations (9)-(17) hold:

$$
\begin{gathered}
\frac{\bar{\alpha}_{n, k}-\alpha_{n, k}}{\bar{m}_{n, k}-m_{n, k}}=\mu_{n, k}=\frac{\alpha_{n+1, k}-\bar{\alpha}_{n, k}}{m_{n+1, k}-\bar{m}_{n, k}} ; \\
\frac{\alpha_{n, k} \bar{m}_{n, k}-\bar{\alpha}_{n, k} m_{n, k}}{\bar{m}_{n, k}-m_{n, k}}=\lambda_{n, k}=\frac{\bar{\alpha}_{n, k} m_{n+1, k}-\alpha_{n+1, k} \bar{m}_{n, k}}{m_{n+1, k}-\bar{m}_{n, k}} ; \\
\frac{\bar{\alpha}_{n, k}-\alpha_{n, k+1}}{\bar{m}_{n, k}-m_{n, k+1}}=\mu_{n, k+1}=\frac{\alpha_{n+1, k+1}-\bar{\alpha}_{n, k}}{m_{n+1, k+1}-\bar{m}_{n, k}} ;
\end{gathered}
$$




$$
\begin{gathered}
\frac{\alpha_{n, k+1} \bar{m}_{n, k}-\bar{\alpha}_{n, k} m_{n, k+1}}{\bar{m}_{n, k}-m_{n, k+1}}=\lambda_{n, k+1}=\frac{\bar{\alpha}_{n, k} m_{n+1, k+1}-\alpha_{n+1, k+1} \bar{m}_{n, k}}{m_{n+1, k+1}-\bar{m}_{n, k}} . \\
\left(\frac{1-s_{k}^{n-1}}{s_{k-1}^{n-1}}\right) \frac{1}{\alpha_{n+1, k}}-\left(\frac{1-s_{k}^{n}}{s_{k-1}^{n}}\right) \frac{1}{\alpha_{n, k}}=\left(\frac{k+1}{n k s_{k-1}^{n-1} s_{k-1}^{n}}\right) \frac{1}{\bar{\alpha}_{n, k}} ; \\
\left(\frac{1-s_{k}^{n-1}}{s_{k-1}^{n-1}}\right) \frac{m_{n+1, k}}{\alpha_{n+1, k}}-\left(\frac{1-s_{k}^{n}}{s_{k-1}^{n}}\right) \frac{m_{n, k}}{\alpha_{n, k}}=\left(\frac{k+1}{n k s_{k-1}^{n-1} s_{k-1}^{n}}\right) \frac{\bar{m}_{n, k}}{\bar{\alpha}_{n, k}} \\
\frac{m_{n+1, k}-\bar{m}_{n, k}}{m_{n+1, k}-m_{n, k}}=\frac{\alpha_{n+1, k}-\bar{\alpha}_{n, k}}{\alpha_{n+1, k}-\alpha_{n, k}}=\frac{n k \bar{\alpha}_{n, k} s_{k-1}^{n-1}\left(s_{k}^{n}-1\right)}{\alpha_{n, k}(k+1)} ; \\
\frac{\bar{m}_{n, k}-m_{n, k}}{m_{n+1, k}-m_{n, k}}=1-\frac{m_{n+1, k}-\bar{m}_{n, k}}{m_{n+1, k}-m_{n, k}}=\frac{n k \bar{\alpha}_{n, k} s_{k-1}^{n}\left(1-s_{k}^{n-1}\right)}{\alpha_{n+1, k}(k+1)} ; \\
-\frac{\lambda_{n, k}}{\mu_{n, k}}=\frac{n s_{k-1}^{n-1} F_{n}^{n+1}-F_{k}^{n}}{n s_{k-1}^{n-1}-(n-k)} .
\end{gathered}
$$

Proof. It is sufficient to prove just one side of the relations (9)-(12) in each case. For example, for (9) think of the slopes of the lines joining the points $\left(m_{n, k}, \alpha_{n, k}\right),\left(\bar{m}_{n, k}, \bar{\alpha}_{n, k}\right)$ and $\left(m_{n+1, k}, \alpha_{n+1, k}\right)$. To prove (9) and (10), first note that

$$
-\frac{\lambda_{n, k}}{\mu_{n, k}}=\frac{\left(m_{n+1, k} / \alpha_{n+1, k}\right)-\left(m_{n, k} / \alpha_{n, k}\right)}{\left(1 / \alpha_{n+1, k}\right)-\left(1 / \alpha_{n, k}\right)}=\frac{\left(\bar{m}_{n, k} / \bar{\alpha}_{n, k}\right)-\left(m_{n, k} / \alpha_{n, k}\right)}{\left(1 / \bar{\alpha}_{n, k}\right)-\left(1 / \alpha_{n, k}\right)}
$$

which follows by observing that

$$
\frac{1}{\bar{\alpha}_{n, k}}-\frac{1}{\alpha_{n, k}}=\frac{\left(1-s_{k}^{n-1}\right)\left(n s_{k-1}^{n-1}-(n-k)\right)}{(k+1) s_{k-1}^{n-1}}=\frac{n k s_{k-1}^{n}\left(1-s_{k}^{n-1}\right)}{k+1}\left[\frac{1}{\alpha_{n+1, k}}-\frac{1}{\alpha_{n, k}}\right]
$$

and

$$
\begin{aligned}
\frac{\bar{m}_{n, k}}{\bar{\alpha}_{n, k}}-\frac{m_{n, k}}{\alpha_{n, k}} & =\frac{\left(1-s_{k}^{n-1}\right)}{k+1}\left(n F_{n}^{n+1}-\frac{F_{k}^{n}}{s_{k-1}^{n-1}}\right) \\
& =\frac{n k s_{k-1}^{n}\left(1-s_{k}^{n-1}\right)}{k+1}\left[\frac{m_{n+1, k}}{\alpha_{n+1, k}}-\frac{m_{n, k}}{\alpha_{n, k}}\right] .
\end{aligned}
$$

To see (9) (and hence (10)), notice that (18) implies that

$$
\begin{aligned}
\frac{1}{\mu_{n, k}} & =\frac{1}{\alpha_{n, k}}\left[\frac{\left(m_{n+1, k} / \alpha_{n+1, k}\right)-\left(m_{n, k} / \alpha_{n, k}\right)}{\left(1 / \alpha_{n, k}\right)-\left(1 / \alpha_{n+1, k}\right)}\right]+\frac{m_{n, k}}{\alpha_{n, k}} \\
& =\frac{1}{\alpha_{n, k}}\left[\frac{\left(\bar{m}_{n, k} / \bar{\alpha}_{n, k}\right)-\left(m_{n, k} / \alpha_{n, k}\right)}{\left(1 / \alpha_{n, k}\right)-\left(1 / \bar{\alpha}_{n, k}\right)}\right]+\frac{m_{n, k}}{\alpha_{n, k}} \\
& =\frac{\bar{m}_{n, k}-m_{n, k}}{\bar{\alpha}_{n, k}-\alpha_{n, k}} .
\end{aligned}
$$


The relations (11) and (12) are derived in an identical manner after proving that

$$
\begin{aligned}
-\frac{\lambda_{n, k+1}}{\mu_{n, k+1}} & =\frac{\left(m_{n+1, k+1} / \alpha_{n+1, k+1}\right)-\left(m_{n, k+1} / \alpha_{n, k+1}\right)}{\left(1 / \alpha_{n+1, k+1}\right)-\left(1 / \alpha_{n, k+1}\right)} \\
& =\frac{\left(\bar{m}_{n, k} / \bar{\alpha}_{n, k}\right)-\left(m_{n, k+1} / \alpha_{n, k+1}\right)}{\left(1 / \bar{\alpha}_{n, k}\right)-\left(1 / \alpha_{n, k+1}\right)}
\end{aligned}
$$

which comes from the calculations

$$
\frac{1}{\bar{\alpha}_{n, k}}-\frac{1}{\alpha_{n, k+1}}=\frac{\left(s_{k}^{n-1}-1\right)\left(n-k-n s_{k}^{n}\right)}{(k+1) s_{k}^{n-1}}=n s_{k}^{n}\left(1-s_{k}^{n-1}\right)\left[\frac{1}{\alpha_{n+1, k+1}}-\frac{1}{\alpha_{n, k+1}}\right]
$$

and

$$
\begin{aligned}
\frac{\bar{m}_{n, k}}{\bar{\alpha}_{n, k}}-\frac{m_{n, k+1}}{\alpha_{n, k+1}} & =\frac{\left(1-s_{k}^{n-1}\right)}{k+1}\left(n F_{n}^{n+1}-\frac{F_{k+1}^{n}}{s_{k}^{n-1}}\right) \\
& =n s_{k}^{n}\left(1-s_{k}^{n-1}\right)\left[\frac{m_{n+1, k+1}}{\alpha_{n+1, k+1}}-\frac{m_{n, k+1}}{\alpha_{n, k+1}}\right] .
\end{aligned}
$$

The identities (13) - (16) may be obtained from direct calculation from the definitions (4) and (5).

3. The optimal $Q$-strategy. For each pair $n, k, 1 \leq k<n$ and $1 \leq j \leq k+1$, define

$$
q_{j}^{n, k}=\frac{\lambda_{n, k}+\mu_{n, k} F_{j-1}^{j}}{1-s_{j-1} \lambda_{n, k}-\mu_{n, k}\left(\sum_{i=1}^{j-2}(f(i) /(i+1))+f(j-1)\right)},
$$

where an empty sum is zero, and define the strategy $\mathbf{q}^{n, k}=\left(q_{1}^{n, k}, q_{2}^{n, k}, \ldots, q_{k}^{n, k}, 1,1, \cdots\right)$.

Using these strategies, the minimax-optimal $Q$-strategies $\mathbf{q}_{M}$ appearing in (2) can now be given.

DEFINITIONS. For all $M>f(1)$,

$$
\begin{aligned}
n(M) & =n \quad \begin{aligned}
\text { when } & m_{n} \leq M<m_{n+1}
\end{aligned} \\
k(M) & =\left\{\begin{array}{ll}
k_{n} & \text { if } m_{n} \leq M<m_{n+1} \text { and } k_{n+1}=k_{n}, \text { or } \\
& \text { if } m_{n} \leq M<m_{n} \text { and } k_{n+1}=k_{n}+1, \\
k_{n+1} & \text { if } \bar{m}_{n} \leq M<m_{n+1} \text { and } k_{n+1}=k_{n}+1 ;
\end{array}\right. \text { and } \\
\mathbf{q}_{M} & =\mathbf{q}^{n(M), k(M)} .
\end{aligned}
$$

Example 3.1. For the expected-value case $f(i) \equiv i$, it follows from the calculations in Example 2.3 that

$$
\begin{gathered}
\mathbf{q}^{3,1}=\left(\frac{7}{13}, 1,1, \cdots\right), \\
\mathbf{q}^{4,1}=\left(\frac{105}{213}, 1,1, \cdots\right), \quad \text { and } \\
\mathbf{q}^{4,2}=\left(\frac{50}{107}, \frac{14}{19}, 1,1, \cdots\right),
\end{gathered}
$$

and together these yield the minimax-optimal $\mathbf{q}_{M}$ in Example 1.2. 
First it must be shown that $\mathbf{q}^{n, k} \in \mathbf{Q}$ for all $1 \leq k<n$; that is, each coordinate must be shown to be a probability.

LEMMA 3.2. For $1 \leq j \leq k+1 \leq n, 0 \leq q_{j}^{n, k} \leq 1$.

Proof. First, the numerator of $q_{j}^{n, k}$ is $>0$. This is because $\lambda_{n, k}+\mu_{n, k} F_{j-1}^{j}=$

$$
\begin{aligned}
& \frac{\alpha_{n, k} \alpha_{n+1, k}}{m_{n+1, k}-m_{n, k}}\left[\frac{m_{n+1, k}}{\alpha_{n+1, k}}-\frac{m_{n, k}}{\alpha_{n, k}}+\left(\frac{1}{\alpha_{n, k}}-\frac{1}{\alpha_{n+1, k}}\right) F_{j-1}^{j}\right] \\
= & \frac{\alpha_{n, k} \alpha_{n+1, k}}{m_{n+1, k}-m_{n, k}}\left[\frac{n-k+1}{k s_{k-1}^{n}}\left(\frac{F_{k}^{n+1}}{n-k+1}-F_{j-1}^{j}\right)-\frac{n-k}{k s_{k-1}^{n-1}}\left(\frac{F_{k}^{n}}{n-k}-F_{j-1}^{j}\right)\right]>0,
\end{aligned}
$$

by Lemma 2.2 (iv) and (v) and the fact that $F_{k}^{n} /(n-k)$ exceeds $F_{j-1}^{j}$ for $j \leq k+1$. Denote the denominator of $q_{j}^{n, k}$ in (19) by $\beta_{j}$. Then to show $\beta_{j}>0$, note that

$$
\beta_{j}-\beta_{j+1}=\left(\lambda_{n, k}+\mu_{n, k} F_{j-1}^{j}\right) / j>0,
$$

by (21) for $j \leq k+1$, so it is sufficient to show that $\beta_{k+1}>0$. But now, from (4) and (5) calculate that

$$
\begin{aligned}
\beta_{k+1} & =\frac{\left(1-s_{k} \alpha_{n, k}\right) \alpha_{n+1, k}}{m_{n+1, k}-m_{n, k}}\left[\frac{m_{n+1, k}}{\alpha_{n+1, k}}-\frac{m_{n, k}}{\alpha_{n, k}}+\left(\frac{1}{\alpha_{n, k}}-\frac{1}{\alpha_{n+1, k}}\right) \frac{F_{k}^{n}}{n-k}\right] \\
& =\frac{\left(1-s_{k} \alpha_{n, k}\right)\left(1-s_{k} \alpha_{n+1, k}\right)}{m_{n+1, k}-m_{n, k}}\left[\frac{F_{k}^{n+1}}{n-k+1}-\frac{F_{k}^{n}}{n-k}\right]>0 .
\end{aligned}
$$

To show that $q_{j}^{n, k} \leq 1$ for $j \leq k+1$, note that the statement that $q_{j}^{n, k} \leq 1$ is equivalent to

$$
\gamma_{j}=\lambda_{n, k}\left(1+s_{j-1}\right)+\mu_{n, k}\left(F_{j-1}^{j}+f(j-1)+\sum_{i=1}^{j-2} \frac{f(i)}{i+1}\right) \leq 1 .
$$

Thus it is sufficient to show that $\gamma_{j+1}-\gamma_{j} \geq 0$ for $j \leq k$ and $q_{k+1}^{n, k} \leq 1$. First, using (4) and the expressions for the numerator and denominator from above, showing that

$$
q_{k+1}^{n, k}=\frac{k s_{k-1}^{n-1}\left(F_{k}^{n+1}-(n-k+1) F_{k}^{k+1}\right)-k s_{k-1}^{n}\left(F_{k}^{n}-(n-k) F_{k}^{k+1}\right)}{(n-k) F_{k}^{n+1}-(n-k+1) F_{k}^{n}} \leq 1
$$

is equivalent after rearrangement to showing that

$$
\frac{1-\left[\left(k s_{k-1}^{n}\right) /(n-k+1)\right]}{1-\left[\left(k s_{k-1}^{n-1}\right) /(n-k)\right]} \leq \frac{\left(F_{k}^{n+1} /(n-k+1)\right)-F_{k}^{k+1}}{\left(F_{k}^{n} /(n-k)\right)-F_{k}^{k+1}} .
$$

But by the convexity of $1 / i$ in $i$, the left-hand side of (23) is dominated by $(n-k) /(n-k-1)$, which in turn is dominated by the right-hand side of (23) using Lemma 2.2 (iii). Furthermore,

$$
\gamma_{j+1}-\gamma_{j}=\left[\lambda_{n, k}+\mu_{n, k}\left(j F_{j}^{j+1}-(j-1) F_{j-1}^{j}\right)\right] / j,
$$

and since $F_{j-1}^{j}$ is increasing in $j$ and $\mu_{n, k}<0$, to prove that $\gamma_{j+1} \geq \gamma_{j}$ for $j \leq k$, it is sufficient to show that

$$
-\frac{\lambda_{n, k}}{\mu_{n, k}} \geq k F_{k}^{k+1}-(k-1) F_{k-1}^{k}
$$


From (17),

$$
-\frac{\lambda_{n, k}}{\mu_{n, k}}=\frac{n s_{k-1}^{n-1} F_{n}^{n+1}-F_{k}^{n}}{n s_{k-1}^{n-1}-(n-k)} \geq \frac{(n+k-1) F_{n}^{n+1}-2(k-1)\left(F_{k}^{n} /(n-k)\right)}{n-k+1}
$$

the last inequality because, by the convexity of $1 / i$,

$$
s_{k-1}^{n-1} \leq \frac{1}{2}(n-k)\left(\frac{1}{n}+\frac{1}{k-1}\right)=\frac{(n-k)(n+k-1)}{2 n(k-1)} .
$$

Finally, it may be seen that the right-hand side of (25) exceeds that of (24) using Lemma 2.2 (iii) again.

It is now possible to prove an inequality that will imply that if the $Q$-player uses strategy $\mathbf{q}_{M}$, then it forces the $P$-player to put positive probability mass only on the points $1,2, \ldots, k(M), n(M), n(M)+1$. Recall the definitions of $\mathbf{q}_{M}$ and $\mathbf{v}_{M}$.

PROPOSITION 3.3. For all $M>f(1)$,

$$
V\left(\mathbf{p}, \mathbf{q}_{M}\right) \geq v_{M} \quad \text { for all } \mathbf{p}=\left(p_{1}, p_{2}, \cdots\right) \in \mathcal{P}_{M},
$$

with equality in (26) if and only if $\sum_{j=1}^{\infty} p_{j} f(j)=M$ and $\mathbf{p}$ assigns positive mass only to points in $\{1, \ldots, k(M), n(M), n(M)+1\}$.

Proof. By the definitions of $\mathbf{q}_{M}$ and $v_{M}$, it is enough to show

$$
V\left(\mathbf{p}, \mathbf{q}^{n, k}\right) \geq \lambda_{n, k}+\mu_{n, k} M, \quad \text { for all } \mathbf{p}=\left(p_{1}, p_{2}, \cdots\right) \in \mathcal{P}_{M},
$$

with equality if and only if $\sum_{j=1}^{\infty} p_{j} f(j)=M$ and $\mathbf{p}$ assigns positive mass only to points $\{1, \ldots, k, n, n+1\}$. First observe that

$$
\prod_{m=1}^{j}\left(1-\frac{q_{m}^{n, k}}{m}\right)=1-s_{j} \lambda_{n, k}-\mu_{n, k}\left(\sum_{m=1}^{j-1} \frac{f(m)}{m+1}+f(j)\right)
$$

and

$$
\sum_{i=1}^{j} q_{i}^{n, k} \prod_{m=1}^{i-1}\left(1-\frac{q_{m}^{n, k}}{m}\right)=j \lambda_{n, k}+j f(j) \mu_{n, k}
$$

For strategies $\mathbf{p}=\left(p_{1}, p_{2}, \cdots\right)$ and $\mathbf{q}=\left(q_{1}, q_{2}, \cdots\right)$, recall (1) and define the Lagrangian

(30)

$$
\begin{aligned}
L(\mathbf{p}, \mathbf{q}) & =V(\mathbf{p}, \mathbf{q})+\lambda_{n, k}\left(1-\sum_{j=1}^{\infty} p_{j}\right)+\mu_{n, k}\left(M-\sum_{j=1}^{\infty} p_{j} f(j)\right) \\
& =\lambda_{n, k}+\mu_{n, k} M+\sum_{j=1}^{\infty} \frac{p_{j}}{j}\left[\sum_{i=1}^{j} q_{i} \prod_{m=1}^{i-1}\left(1-\frac{q_{m}}{m}\right)-\lambda_{n, k} j-\mu_{n, k} j f(j)\right] .
\end{aligned}
$$

The dependence of $L$ on $n$ and $k$ will be suppressed in the notation. For $\mathbf{p} \in \mathcal{P}_{M}$, since $\mu_{n, k}<$ 0 , it is immediate that $V(\mathbf{p}, \mathbf{q}) \geq L(\mathbf{p}, \mathbf{q})$, with equality if and only if $\sum_{j=1}^{\infty} p_{j} f(j)=M$. When $\mathbf{q}=\mathbf{q}^{n, k}$ it is now sufficient to show that the coefficient of $p_{j} / j$ in (30) is $\geq 0$ for all $j$ and is $=0$ if and only if $j=1,2, \ldots, k, n, n+1$. But for $1 \leq j \leq k$ this is true from (29). 
For $j>k$ the coefficient of $p_{j} / j$ is

$$
\begin{aligned}
\sum_{i=1}^{k} q_{i}^{n, k} \prod_{m=1}^{i-1}\left(1-\frac{q_{m}^{n, k}}{m}\right)+\prod_{m=1}^{k}\left(1-\frac{q_{m}^{n, k}}{m}\right) \sum_{i=k+1}^{j} \prod_{r=k+1}^{i-1}\left(\frac{r-1}{r}\right) \\
-\lambda_{n, k} j-\mu_{n, k} j f(j)=\lambda_{n, k}(k-j)-\mu_{n, k} F_{k}^{j}+k s_{k-1}^{j-1} \\
\cdot\left[1-s_{k} \lambda_{n, k}-\mu_{n, k}\left(\sum_{i=1}^{k-1} \frac{f(i)}{i+1}+f(k)\right)\right],
\end{aligned}
$$

from (29), with the convention that an empty product is 1 . Using (4)-(6) and (22) and putting $G_{s}^{r}=F_{s}^{r} /(r-s)$ for $r>s,(31)$ reduces to

$$
\frac{\left(1-s_{k} \alpha_{n, k}\right)\left(1-s_{k} \alpha_{n+1, k}\right)(j-k) k \delta_{j}}{m_{n+1, k}-m_{n, k}}
$$

where

$$
\delta_{j}=\frac{s_{k-1}^{j-1}}{j-k}\left(G_{k}^{n+1}-G_{k}^{n}\right)-\frac{s_{k-1}^{n-1}}{n-k}\left(G_{k}^{n+1}-G_{k}^{j}\right)+\frac{s_{k-1}^{n}}{n-k+1}\left(G_{k}^{n}-G_{k}^{j}\right) .
$$

Note that $\delta_{n}=\delta_{n+1}=0$, and

$$
\delta_{j+1}-\delta_{j}=\left(\frac{s_{k-1}^{j}}{j-k+1}-\frac{s_{k-1}^{j-1}}{j-k}\right)\left(G_{k}^{n+1}-G_{k}^{n}\right)-\left(\frac{s_{k-1}^{n}}{n-k+1}-\frac{s_{k-1}^{n-1}}{n-k}\right)\left(G_{k}^{j+1}-G_{k}^{j}\right) .
$$

But, using Lemma 2.1 (ii), $G_{k}^{j+1}-G_{k}^{j}$ is positive and nondecreasing and the expression $\left(s_{k-1}^{j} /(j-k+1)\right)-\left(s_{k-1}^{j-1} /(j-k)\right)$ is negative and increasing in $j>k$; hence $\delta_{j+1}<\delta_{j}$ for $j<n$ and $\delta_{j+1}>\delta_{j}$ for $j>n$, which shows that (31) is $>0$ when $j>k, j \neq n, n+1$, which completes the proof.

Once the analogue of Proposition 3.3 is proved for the $P$-strategy (Proposition 4.2 below), this will establish the minimax-optimality of both $\mathbf{q}_{M}$ and $\mathbf{p}_{M}$ and that $v_{M}$ is the value of the game.

4. The optimal $\boldsymbol{P}$-strategy and main theorem. For $1 \leq k \leq k_{n}<n$, define distributions $\mathbf{p}^{n, k}=\left(p_{1}^{n, k}, p_{2}^{n, k}, \cdots\right)$ concentrated on the points $\{1,2, \ldots, k, n\}$ by

$$
p_{j}^{n, k}= \begin{cases}\alpha_{n, k} /(j+1) & \text { for } 1 \leq j<k \\ \alpha_{n, k}\left(s_{k-1}^{n-1}-1\right) / s_{k-1}^{n-1} & \text { for } j=k \\ n \alpha_{n, k} /\left(k s_{k-1}^{n-1}\right) & \text { for } j=n\end{cases}
$$

with $p_{j}^{n, k} \equiv 0$ otherwise. Note that $\mathbf{p}^{n, k}$ is a distribution only for $k \leq k_{n}$ and that $m_{n, k}=$ $\sum_{j=1}^{\infty} f(j) p_{j}^{n, k} . \operatorname{Set}^{n}=\mathbf{p}^{n, k_{n}}$ for $n \geq 2$, and $\mathbf{p}^{1}=(1,0,0, \cdots)$. Also, when $k_{n+1}=k_{n}+1$, define the distribution $\overline{\mathbf{p}}^{n}=\left(\bar{p}_{1}^{n}, \bar{p}_{2}^{n}, \cdots\right)$ concentrated on $\left\{1,2, \ldots, k_{n}, n, n+1\right\}$ by

$$
\bar{p}_{j}^{n}= \begin{cases}\bar{\alpha}_{n} /(j+1) & \text { for } 1 \leq j \leq k_{n} \\ \bar{\alpha}_{n} n^{2}\left(s_{k_{n}}^{n}-1\right) /\left(k_{n}+1\right) & \text { for } j=n \\ \bar{\alpha}_{n} n(n+1)\left(1-s_{k_{n}}^{n-1}\right) /\left(k_{n}+1\right) & \text { for } j=n+1\end{cases}
$$

with $\bar{p}_{j}^{n} \equiv 0$ otherwise. Note that $\bar{m}_{n}=\sum_{j=1}^{\infty} f(j) \bar{p}_{j}^{n}$.

Remark. The distributions $\mathbf{p}^{n}, n=1,2, \cdots$ are exactly the minimax-optimal $P$-player strategies for the $N \leq n$ problem studied in Hill and Krengel (1991). 
The minimax-optimal (worst-case distribution) strategy $\mathbf{p}_{M}$ in (2) for player $P$ can now be shown to be convex combinations of these base strategies $\mathbf{p}^{n}$ and $\overline{\mathbf{p}}^{n}$.

DEFINITION. For all $M>f(1)$, define $\mathbf{p}_{M} \in \mathcal{P}_{M}$ by

$$
\mathbf{p}_{M}=\left\{\begin{array}{l}
\left(\frac{m_{n+1}-M}{m_{n+1}-m_{n}}\right) \mathbf{p}^{n}+\left(\frac{M-m_{n}}{m_{n+1}-m_{n}}\right) \mathbf{p}^{n+1} \in \mathcal{P}_{M} \\
\text { if } k_{n+1}=k_{n} \text { and } m_{n} \leq M<m_{n+1} \\
\left(\frac{\bar{m}_{n}-M}{\bar{m}_{n}-m_{n}}\right) \mathbf{p}^{n}+\left(\frac{M-m_{n}}{\bar{m}_{n}-m_{n}}\right) \overline{\mathbf{p}}^{n} \in \mathcal{P}_{M} \\
\text { if } k_{n+1}=k_{n}+1 \text { and } m_{n} \leq M<\bar{m}_{n} \\
\left(\frac{m_{n+1}-M}{m_{n+1}-\bar{m}_{n}}\right) \overline{\mathbf{p}}^{n}+\left(\frac{M-\bar{m}_{n}}{m_{n+1}-\bar{m}_{n}}\right) \mathbf{p}^{n+1} \in \mathcal{P}_{M} \\
\text { if } k_{n+1}=k_{n}+1 \text { and } \bar{m}_{n} \leq M<m_{n+1} .
\end{array}\right.
$$

Notice that the strategy $\mathbf{p}_{M}$ places positive probability mass only on the points in the set $\{1, \ldots, k(M), n(M), n(M)+1\}$ and that, when $k_{n+1}=k_{n}, \bar{m}_{n}$ is a changeover point in that when $M$ increases through the value $M=\bar{m}_{n}, \mathbf{p}_{M}$ increases the number of points in its support by 1.

Example 4.1. For the expected-value case $f(i) \equiv i$, it follows from the calculations in Example 2.3 that

$$
\begin{gathered}
\mathbf{p}^{3}=\mathbf{p}^{3,1}=\left(\frac{1}{7}, 0, \frac{6}{7}, 0,0, \cdots\right), \\
\mathbf{p}^{4}=\mathbf{p}^{4,1}=\left(\frac{5}{29}, 0,0, \frac{24}{29}, 0,0, \cdots\right), \\
\overline{\mathbf{p}}^{4}=\left(\frac{3}{17}, 0,0, \frac{4}{17}, \frac{10}{17}, 0,0, \cdots\right), \quad \text { and } \\
\mathbf{p}^{5}=\mathbf{p}^{5,2}=\left(\frac{13}{75}, \frac{2}{75}, 0,0, \frac{60}{75}, 0,0, \cdots\right),
\end{gathered}
$$

and together these yield the minimax-optimal strategies $\mathbf{p}_{M}$ in Example 1.2.

Now the analogue of Proposition 3.3 will be proved, which, together with Proposition 3.3, will establish the minimax-optimality of $\mathbf{q}_{M}$ and $\mathbf{p}_{M}$ simultaneously.

PROPOSITION 4.2. For all $M>f(1)$,

$$
V\left(\mathbf{p}_{M}, \mathbf{q}\right) \leq v_{M} \quad \text { for all } \mathbf{q}=\left(q_{1}, q_{2}, \cdots\right) \in Q .
$$

Proof. The argument of Theorem C of Hill and Krengel (1991) demonstrates that

$$
V\left(\mathbf{p}^{n, k},\left(q_{1}, q_{2}, \ldots, q_{k}, 1,1, \cdots\right)\right) \equiv \alpha_{n, k}, \quad \text { for } 0 \leq q_{i} \leq 1, i=1, \ldots, k,
$$

and a similar argument shows that, when $k_{n+1}=k_{n}+1$,

$$
V\left(\overline{\mathbf{p}}^{n},\left(q_{1}, q_{2}, \ldots, q_{k_{n+1}}, 1,1, \cdots\right)\right) \equiv \bar{\alpha}_{n}, \quad \text { for } 0 \leq q_{i} \leq 1, i=1, \ldots, k_{n+1} .
$$

Furthermore, Hill and Krengel (1991) established that for any $\mathbf{q}=\left(q_{1}, q_{2}, \cdots\right) \in \mathcal{Q}$ and $k \geq k_{n}$,

(34) $V\left(\mathbf{p}^{n}, \mathbf{q}\right) \leq V\left(\mathbf{p}^{n},\left(q_{1}, \ldots, q_{k}, 1,1, \cdots\right)\right) \leq V\left(\mathbf{p}^{n},\left(q_{1}, \ldots, q_{k_{n}}, 1,1, \cdots\right)\right)=\alpha_{n}$, 
and again it is straightforward to prove that when $k_{n+1}=k_{n}+1$ and $k \geq k_{n+1}$,

(35) $V\left(\overline{\mathbf{p}}^{n}, \mathbf{q}\right) \leq V\left(\overline{\mathbf{p}}^{n},\left(q_{1}, \ldots, q_{k}, 1,1, \cdots\right)\right) \leq V\left(\overline{\mathbf{p}}^{n},\left(q_{1}, \ldots, q_{k_{n+1}}, 1,1, \cdots\right)\right)=\bar{\alpha}_{n}$.

Three cases corresponding to the three possibilities in (32) must be considered.

Case 1. $k_{n+1}=k_{n}$ and $m_{n} \leq M<m_{n+1}$.

$$
\begin{aligned}
V\left(\mathbf{p}_{M}, \mathbf{q}\right) & =\left(\frac{m_{n+1}-M}{m_{n+1}-m_{n}}\right) V\left(\mathbf{p}^{n}, \mathbf{q}\right)+\left(\frac{M-m_{n}}{m_{n+1}-m_{n}}\right) V\left(\mathbf{p}^{n+1}, \mathbf{q}\right) \\
& \leq\left(\frac{m_{n+1}-M}{m_{n+1}-m_{n}}\right) \alpha_{n}+\left(\frac{M-m_{n}}{m_{n+1}-m_{n}}\right) \alpha_{n+1}=\lambda_{n, k_{n}}+\mu_{n, k_{n}} M,
\end{aligned}
$$

for all $\mathbf{q} \in \mathcal{Q}$, using (34).

Case 2. $k_{n+1}=k_{n}+1$ and $m_{n} \leq M<\bar{m}_{n}$. Using the relations in (9)-(16) and setting $M=\bar{m}_{n}$ it may be seen that $\mathbf{p}_{\bar{m}_{n}}=\overline{\mathbf{p}}_{n}$, whence

$$
\mathbf{p}_{M} \equiv\left(\frac{m_{n+1, k_{n}}-M}{m_{n+1, k_{n}}-m_{n}}\right) \mathbf{p}^{n}+\left(\frac{M-m_{n}}{m_{n+1, k_{n}}-m_{n}}\right) \mathbf{p}^{n+1, k_{n}}
$$

Note from Lemma 2.4 (viii) that for $M$ in this range, $M \leq m_{n+1, k_{n}}$. For any $\mathbf{q} \in \mathcal{Q}$,

$$
V\left(\mathbf{p}_{M}, \mathbf{q}\right)=\left(\frac{m_{n+1, k_{n}}-M}{m_{n+1, k_{n}}-m_{n}}\right) V\left(\mathbf{p}^{n}, \mathbf{q}\right)+\left(\frac{M-m_{n}}{m_{n+1, k_{n}}-m_{n}}\right) V\left(\mathbf{p}^{n+1, k_{n}}, \mathbf{q}\right),
$$

which, by (34), is dominated by

$$
\begin{aligned}
&\left(\frac{m_{n+1, k_{n}}-M}{m_{n+1, k_{n}}-m_{n}}\right) V\left(\mathbf{p}^{n},\left(q_{1}, \ldots, q_{k_{n}+1}, 1,1, \cdots\right)\right) \\
&+\left(\frac{M-m_{n}}{m_{n+1, k_{n}}-m_{n}}\right) V\left(\mathbf{p}^{n+1, k_{n}},\left(q_{1}, \ldots, q_{k_{n}+1}, 1,1, \cdots\right)\right) .
\end{aligned}
$$

Now consider the coefficient of $q_{k_{n}+1} \prod_{m=1}^{k_{n}}\left(1-q_{m} / m\right)$ in (36) which equals

$$
\begin{aligned}
& \frac{p_{n}^{n, k_{n}}}{n}\left(\frac{m_{n+1, k_{n}}-M}{m_{n+1, k_{n}}-m_{n}}\right)\left(1-s_{k_{n}}^{n-1}\right)+\frac{p_{n+1}^{n+1, k_{n}}}{n+1}\left(\frac{M-m_{n}}{m_{n+1, k_{n}}-m_{n}}\right)\left(1-s_{k_{n}}^{n}\right) \\
= & \frac{\alpha_{n}}{k_{n}}\left(\frac{m_{n+1, k_{n}}-M}{m_{n+1, k_{n}}-m_{n}}\right)\left(\frac{1-s_{k_{n}}^{n-1}}{s_{k_{n}-1}^{n-1}}\right)+\frac{\alpha_{n+1, k_{n}}}{k_{n}}\left(\frac{M-m_{n}}{m_{n+1, k_{n}}-m_{n}}\right)\left(\frac{1-s_{k_{n}}^{n}}{s_{k_{n}-1}^{n}}\right) .
\end{aligned}
$$

Rearranging this expression as

$$
\frac{\alpha_{n} \alpha_{n+1, k_{n}}}{k_{n}\left(m_{n+1, k_{n}}-m_{n}\right)}\left[\left(\frac{1-s_{k_{n}}^{n-1}}{s_{k_{n}-1}^{n-1}}\right)\left(\frac{m_{n+1, k_{n}}-M}{\alpha_{n+1, k_{n}}}\right)+\left(\frac{1-s_{k_{n}}^{n}}{s_{k_{n}-1}^{n}}\right)\left(\frac{M-m_{n}}{\alpha_{n}}\right)\right],
$$

and using (13) and (14) (with $k=k_{n}$ ) this equals

$$
\frac{\alpha_{n} \alpha_{n+1, k_{n}}\left(k_{n}+1\right)}{n k_{n} s_{k_{n}-1}^{n-1} s_{k_{n}-1}^{n}\left(m_{n+1, k_{n}}-m_{n}\right)}\left(\frac{\bar{m}_{n}-M}{\bar{\alpha}_{n}}\right) \geq 0,
$$

for $M \leq \bar{m}_{n}$. It follows that (36) is not decreased by taking $q_{k_{n}+1}=1$, so it may be seen that

$$
\begin{aligned}
V\left(\mathbf{p}_{M}, \mathbf{q}\right) \leq\left(\frac{m_{n+1, k_{n}}-M}{m_{n+1, k_{n}}-m_{n}}\right) & V\left(\mathbf{p}^{n},\left(q_{1}, \ldots, q_{k_{n}}, 1,1, \cdots\right)\right) \\
& +\left(\frac{M-m_{n}}{m_{n+1, k_{n}}-m_{n}}\right) V\left(\mathbf{p}^{n+1, k_{n}},\left(q_{1}, \ldots, q_{k_{n}}, 1,1, \ldots\right)\right),
\end{aligned}
$$


which in turn implies that

$$
V\left(\mathbf{p}_{M}, \mathbf{q}\right) \leq\left(\frac{m_{n+1, k_{n}}-M}{m_{n+1, k_{n}}-m_{n}}\right) \alpha_{n}+\left(\frac{M-m_{n}}{m_{n+1, k_{n}}-m_{n}}\right) \alpha_{n+1, k_{n}}=\lambda_{n, k_{n}}+\mu_{n, k_{n}} M .
$$

Case 3. $k_{n+1}=k_{n}+1$ and $\bar{m}_{n} \leq M<m_{n+1}$. It follows from (34) and (35) as in Case 1 that

$$
\begin{aligned}
V\left(\mathbf{p}_{M}, \mathbf{q}\right) & =\left(\frac{m_{n+1}-M}{m_{n+1}-\bar{m}_{n}}\right) V\left(\overline{\mathbf{p}}^{n}, \mathbf{q}\right)+\left(\frac{M-\bar{m}_{n}}{m_{n+1}-\bar{m}_{n}}\right) V\left(\mathbf{p}^{n+1}, \mathbf{q}\right) \\
& \leq\left(\frac{m_{n+1}-M}{m_{n+1}-\bar{m}_{n}}\right) \bar{\alpha}_{n}+\left(\frac{M-\bar{m}_{n}}{m_{n+1}-\bar{m}_{n}}\right) \alpha_{n+1} \\
& =\lambda_{n, k_{n+1}}+\mu_{n, k_{n+1}} M,
\end{aligned}
$$

using (11) and (12).

The main results in this paper can now be summarized in the following theorem.

THEOREM 4.3. With $\mathbf{p}_{M}, \mathbf{q}_{M}$, and $v_{M}$ as in (32), (20), and (7), respectively,

$$
V\left(\mathbf{p}_{M}, \mathbf{q}\right) \leq V\left(\mathbf{p}_{M}, \mathbf{q}_{M}\right)=v_{M} \leq V\left(\mathbf{p}, \mathbf{q}_{M}\right), \quad \text { for all } \mathbf{p} \in \mathcal{P}_{M}, \mathbf{q} \in \mathcal{Q} .
$$

Proof. The proof is immediate from Propositions 3.3 and 4.2 and the definitions.

Acknowledgment. The main question addressed in this paper was raised by Shmuel Gal in the AMS-IMS-SIAM Conference on Sequential Search Strategies in Amherst (1990), and the authors are grateful to the organizers Tom Ferguson and Steve Samuels for invitations to speak at that conference.

\section{REFERENCES}

[1] T. S. Ferguson, Who solved the secretary problem? Statist. Sci., 4 (1989), pp. 282-296.

[2] P. R. FreEman, The secretary problem and its extensions: A review, Internat. Statist. Rev., 51 (1983), pp. $189-206$.

[3] T. P. Hill and U. Krengel, Minimax-optimal stop rules and distributions in secretary problems, Ann. Probab., 19 (1991), pp. 342-353.

[4] E. Presman and I. Sonin, The best choice problem for a random number of objects, Theory Probab. Appl., 17 (1972), pp. 657-668. 